\title{
Study on the treatment of the p15 gene combined with Bcr-abl-specific siRNA and STI571 for chronic myeloid leukemia
}

\author{
W. Wang, Y. Du, N.-N. Li, F.-F. Lv and G.-Q. Lin \\ Department of Hematology, Southeast Hospital Affiliated to Xiamen University, \\ Zhangzhou, Fujian, China \\ Corresponding author: W. Wang \\ E-mail: wangwei220012@163.com
}

Genet. Mol. Res. 13 (2): 4089-4101 (2014)

Received February 14, 2013

Accepted October 30, 2013

Published May 30, 2014

DOI http://dx.doi.org/10.4238/2014.May.30.4

\begin{abstract}
The aim of this study was to investigate the effect of the p15 gene combined with Bcr-abl-specific siRNA and STI571 on the proliferation, cell cycle and apoptosis of K562 chronic myeloid leukemia cells. Using the gene sequence results, we amplified the p15 gene from normal peripheral blood by RT-PCR, and constructed a p15-pcDNA3.1 vector. The K562 cell line with G418 resistance was screened, synthesized and transfected for bcr-abl gene fusion point for 21-nt siRNA. In p15-pcDNA3.1-K562 cells, the growth rate was slower than that of the control K562 cells, G0/G1-phase was increased and S-phase was decreased significantly. In the siRNA group, bcr-abl fusion gene expression was significantly decreased in K562 cells accompanied by the downregulation of BCL-xL protein expression and G1-phase arrest. Cell survival rate was significantly decreased compared with the sole p15-K562 cell group and the sole RNA interference-K562 cell group. In the combination of p15pcDNA3.1-K562 cells with STI571, the proportion of apoptosis was significantly increased and the cell survival rate was significantly decreased compared with the p15-K562 cell group and STI571-K562
\end{abstract}


cell group. siRNA at $30 \mathrm{pM}$ combined with $0.5 \mu \mathrm{M}$ STI571 promoted apoptosis compared with sole application. The p15 gene combined with siRNA had a synergistic effect on the inhibition of proliferation and the induction of apoptosis in K562 cells. Exogenous p15 protein expression combined with STI571 appeared to have a synergistic effect on proliferation inhibition and apoptosis induction in K562 cells. The combination of low-dose RNA interference with STI571 showed a synergistic effect in inducing apoptosis.

Key words: p15; Cloning expression; siRNA; STI571; Chronic myeloid leukemia

\section{INTRODUCTION}

Chronic myeloid leukemia (CML) is a malignant proliferative disease due to abnormal clones of pluripotent stem cells. About $90 \%$ CML patients have the distinctive $\mathrm{Ph}$ chromosome in their leukemic cells (Helgason et al., 2011; Leonetti et al., 2011), which is formed by the mutual translocation of the 3 zone 4 band in the long arm of chromosome 9 (9q34) and the 1 zone 1 band in the long arm of chromosome 22 (22q11), $\mathrm{t}(9 ; 22)$ (q34; q11). In addition, the proto-oncogene c-abl at 9q34 id broken at the 5'-end of its second exon, and shifted to 22q11 of the bcr gene at the 3'-end of the second or third exon (M-bcr) for rearrangement, forming the fusion gene bcr/abl. The gene encodes a protein of $210 \mathrm{kDa}$, known as the P210 protein. The P210 protein disorders the cell control system with the activity of protein tyrosine kinase, which causes CML with the malignant differentiation and proliferation of the granulocyte series. In other words, the formation of bcr/abl fusion gene is the key to the pathogenesis of CML.

On the basis of the pathogenesis of CML, recent research has focused on the inhibition of bcr-abl fusion gene and p210 of protein tyrosine kinase activity. For the former, the most effective method is RNA interference (Zaree-Mahmodabady et al., 2010), and the specific inhibitor STI571 is used in the case of the latter. Previous studies have shown that RNA interference and STI571 alone have some limitations, such as transfection efficiency, toxicity and drug resistance (Swords et al., 2009; Bengió et al., 2011), especially in the acute transformation phase of CML with more abnormal genes (Yamauchi and Ueda, 2011; Nicolini et al., 2011). In the acute transformation phase, combined treatment is the trend for CML (Druker et al., 2002). The bcr-abl oncogene fusion gene belongs to the scope of the oncogenes, and the inactivation of antioncogenes plays an important role in CML blast crisis. p15, as an anti-oncogene regulating the cell cycle (Güran et al., 1998), showed only abnormal methylation in CML cells (Nguyen et al., 2000). Without further study, the mechanism is still not known in the functional inactivation of the gene and protein for CML. For the treatment of CML at the genetic level, one thing is to inhibit the abnormal bcr-abl fusion gene and protein, another is to sustain anti-oncogenes by gene cloning expression with the effect of tumor suppression. In the present study, we synthesized the specific siRNA for the fusion point of bcr-abl by microRNA interference, inhibited the activity of the $\mathrm{p} 210$ protein by STI571, and restored inactive $\mathrm{p} 15$ by gene cloning expression. Based on the different pathogenesis of CML, these treatments were combined to induce more effective tumor suppression and apoptosis, which was helpful in exploring the mechanism of p15 gene abnormalities in blast crisis, and provide new strategies and methods in gene therapy of CML. 


\section{MATERIAL AND METHODS}

Specific siRNA sequences were from Dhamacon (Pittsburgh, PA, USA). The restriction enzymes EcoRI and HindIII, Taq DNA polymerase, T4 ligase, the reverse transcriptase enzymes, Trizol RNA extraction reagent, and DNA purification reagent were obtained from Invitrogen. p15, cyclin E, bcl-xL, etc., mouse anti-human monoclonal antibody and goat antimouse secondary antibody were purchased from Sigma. Bacterial strains and the plasmid pcDNA3.1 were from Invitrogen. Escherichia coli JM109 strain was supplied by the Department of Biochemistry of the Fourth Military Medical University, Xian, Shaanxi, China.

\section{Cell culture}

K562 cells, were cultured in RPMI 1640 nutrient medium with $10 \%(\mathrm{v} / \mathrm{v})$ fetal bovine serum in a $5 \% \mathrm{CO}_{2}$ incubator.

\section{Primer}

The upstream primer, 5'-gTAAgCTTATggCCACgTCTCTggATTTTA-3', and the downstream primer, 5'-TggAATTCTTAACTACTAgACCAATCTTgA-3', and HindIII and EcoRI restriction sites were chosen. PCR products were about $465 \mathrm{bp}$, and synthesized by the Shanghai Shenggong Company.

\section{RT-PCR}

Total RNA was extracted from peripheral blood mononuclear cells by Trizol, which was reverse-transcribed into cDNA for PCR. The reaction system included $0.2 \mu \mathrm{g}$ cDNA, 0.5 $\mu \mathrm{L} 10 \mathrm{mM}$ dNTPs, 10 pmol primers and $1 \mathrm{U}$ Taq DNA polymerase. The amplification reaction consisted of 30 cycles $\left(94^{\circ} \mathrm{C}\right.$ for $45 \mathrm{~s}, 55^{\circ} \mathrm{C}$ for $40 \mathrm{~s}, 72^{\circ} \mathrm{C}$ for $\left.1 \mathrm{~min}\right)$ and $30 \mathrm{~min}$ of final extension. The PCR product was observed by $1 \%$ agarose gel electrophoresis with ethidium bromide staining in a UV-reflecting projectoscope.

\section{T carrier conjugation and DNA sequencing}

A volume of $5 \mu \mathrm{L}$ recovered products with $1 \mu \mathrm{L}$ T carrier and $4 \mu \mathrm{L}$ quick ligation solution were incubated for $4 \mathrm{~h}$ at $16^{\circ} \mathrm{C}$, and transformed into the JM109 strain. The white colonies were chosen for shaking bacteria and extracting plasmid DNA. The sequencing was carried out by the Shanghai Shenggong Company after the verification of restriction enzyme digestion.

\section{p15-pcDNA3.1 recombinant plasmid and transfection}

PcDNA3.1 and p15 T carrier were digested with HindIII and EcoRI. Ligation was done with T4 ligase, and the plasmid transformed into the JM109 strain. The plasmid was extracted from positive colonies, and transfected to K562 cells by lipoplast. After a 48-h culture, stable transfection was accomplished in 1640 nutrient liquid with $500 \mathrm{mg} / \mathrm{L}$ G418 for screening positive colonies. The positive colonies were cells that survived after 28 days. 


\section{bcr-abl fusion gene of specific siRNA sequence}

According to the method of Elbashir et al. (2001), 75 bp downstream of the initiation codon, the initiation basic group was AA and $\mathrm{G} / \mathrm{C}$ content was $42.8 \%$. After screening using NCBI BLAST, we found that only the b3-a2 type bcr-abl fusion gene cDNA sequence exactly matched the sequence, while others had 3 or more basic group differences. The sequence was as follows, 5'-AAGCAGAGTTCAAAAGCCCTT-3', in which AAGC was the fusion site of the bcr-abl fusion gene. According to the siRNA design, the sense strand sequence was 5'-GCAGAGUUCAAAAGCCCUUdTdT-3', and the anti-sense strand sequence was 5'-AAGGGCUUUUGAACUCUGCdTdT-3'. The luciferase gene (ID M15077, 394 414) was chosen as a non-specific control. Finally, the double-stranded RNA with reannealing was the bcr-abl fusion gene of specific siRNA.

\section{Transfection of specific siRNA}

Rats were divided into four groups, 1) transfection was aimed at the bcr-abl gene fusion position of specific siRNA in the siRNA group, 2) transfection was aimed at luciferase gene siRNA in the independent group, 3) only liposome was controlled in the empty vector group, 4) the control group had no treatment. The Oligofectamine reagent was added to the siRNA transfection medium. To ensure transfection efficiency, K562 cells were chosen with less than five generations and good growth condition in the exponential phase of growth. The cell count was done before the transfection. Cells were washed with DMEM serum-free medium, and diluted to $15 \times 10^{6}$ cells $/ \mathrm{mL}$, seeded at $30 \times 10^{5}$ cells $/$ well on a $24-w e l l$ plate, and the siRNA concentration was $60 \mathrm{pmol} / \mathrm{well}$. Groups 1 and 2 contained a mixture of liposome and siRNA, and group 3 only equivalent liposome. Other processing conditions were identical in the four groups, at $37^{\circ} \mathrm{C}, 5 \% \mathrm{CO}_{2}$ incubation for $4 \mathrm{~h}, 10 \%$ fetal bovine serum added, and $24-96-\mathrm{h}$ culture.

\section{Western blot of bcr-abl, p15 and Bcl-xL protein}

K562 cells were collected, and a cell lysate was prepared for protein extraction, in an ice bath for $20 \mathrm{~min}$. A $20-\mu \mathrm{g}$ aliquot of cleaved product was subjected to denaturing polyacrylamide gel electrophoresis, and then transferred to a nitrocellulose membrane. The specific bcr-abl fusion protein, $\mathrm{p} 15$ protein, Bcl-xL mouse anti-human monoclonal antibodies were the primary antibodies, and peroxidase-labeled sheep anti-mouse IgG was the secondary antibody. The chemical enhancement luminescence method was applied for imaging.

\section{Cell biological activity}

\section{Experimental groups}

1) p15 gene cloning combined with RNA interference experiment: the p15 group, RNA interference group, the combined group, and the empty vector control group.

2) p15 gene cloning combined with STI571 experiment: the p15 group, the STI571 group, the combined group, and the empty vector control group.

3) RNA interference combined with STI571 experiment: the RNA interference group, the STI571 group, the combined group and, the empty vector control group. 


\section{Cell growth index and cell survival rate by MTT assay}

A total of $5 \times 10^{4} / \mathrm{mL}$ cells were seeded on a 96-well plate, and the average number of cells from 3 wells in each group was determined at days 1, 2, 3, 4, and 5 for the cell growth curve and the cell survival rate. The experiments were repeated three times (data not shown).

\section{Analysis of cell cycle and apoptosis by flow cytometry}

The liposomes were transfected into K562 cells, and cells were collected after a 48-h reaction. Cells were washed twice with PBS, and kept in ice-cold $70 \%$ ethanol for $2 \mathrm{~h}$ or more. Analysis was performed by flow cytometry after the extraction process with sodium phosphate-sodium citrate buffer, RNase A digestion and a 30-min staining with propidium iodide.

\section{Statistical analysis}

The SPSS16.0 statistical software was used for data analysis. The repeated measures ANOVA was used in group comparisons, and the Dunnett $t$-test was used in comparison of two sets.

\section{RESULTS}

\section{RT-PCR amplification of p15}

The full-length cDNA sequence of the $\mathrm{p} 15$ gene was amplified from peripheral blood mononuclear cell by RT-PCR, for building the p15-pcDNA3.1 plasmid vector. The product was detected by $1 \%$ agarose gel electrophoresis, showing a positive band of about $465 \mathrm{bp}$, which matched the p15 gene full-length cDNA sequence. Meanwhile, the product from K562 cells amplified by the primer was the same size as the p15 gene fragment (Figure 1).

\section{Recombinant plasmid}

p15 gene full-length cDNA sequence from peripheral blood mononuclear cells and pcDNA3.1 plasmids were digested with HindIII and EcoRI, with sticky ends connected by T4 ligase, constructing the p15-pcDNA3.1 recombinant plasmid. The recombinant plasmid was identified with HindIII and EcoRI restriction enzyme digestion, where the DNA fragment was about $465 \mathrm{bp}$, the same size as the p15 gene full-length cDNA sequence (Figure 2).

\section{p15 gene sequence analysis}

p15 gene full-length cDNA sequence from peripheral blood mononuclear cells and puc18-T sequencing vector were digested with HindIII and EcoRI, with sticky end connected by $\mathrm{T} 4$ ligase, constructing the $\mathrm{p} 15$-puc18-T recombination $\mathrm{T}$ vector. The $\mathrm{p} 15$ gene connected with $T$ vector was sequenced with the universal primer. The sequencing result was indexed with the BLAST program, which confirmed that the sequencing result of the p15 gene from peripheral blood mononuclear cells was identical with the p15 gene in GenBank. 


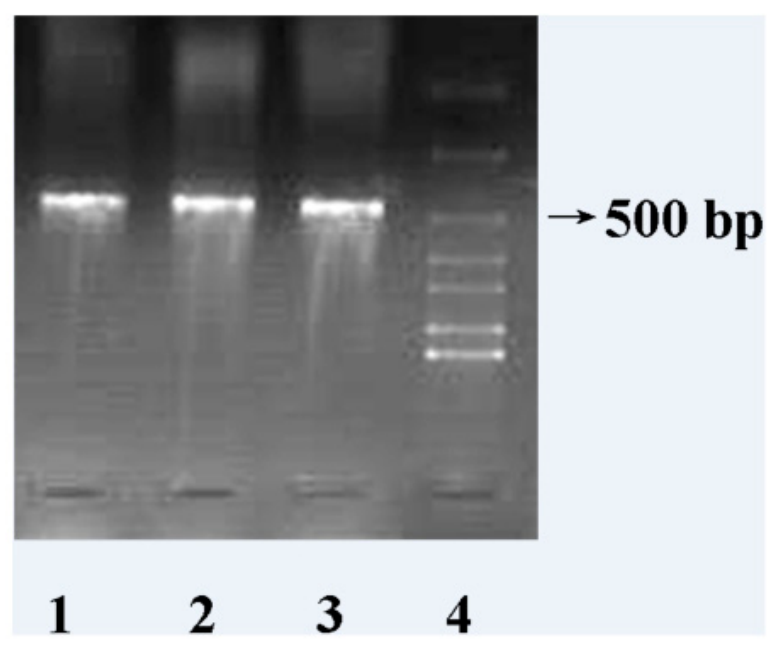

Figure 1. DNA fragment amplified from peripheral blood mononuclear cell (PBMC) and K562 by RT-PCR. Lane 1 $=$ DNA fragment amplified from PMC (about $465 \mathrm{bp}$ ); lane $2=$ control of P15 gene (stomach tissue); lane $3=$ DNA fragment amplified from K562 cells (about 465 bp); lane 4 = DGL2000 DNA marker.

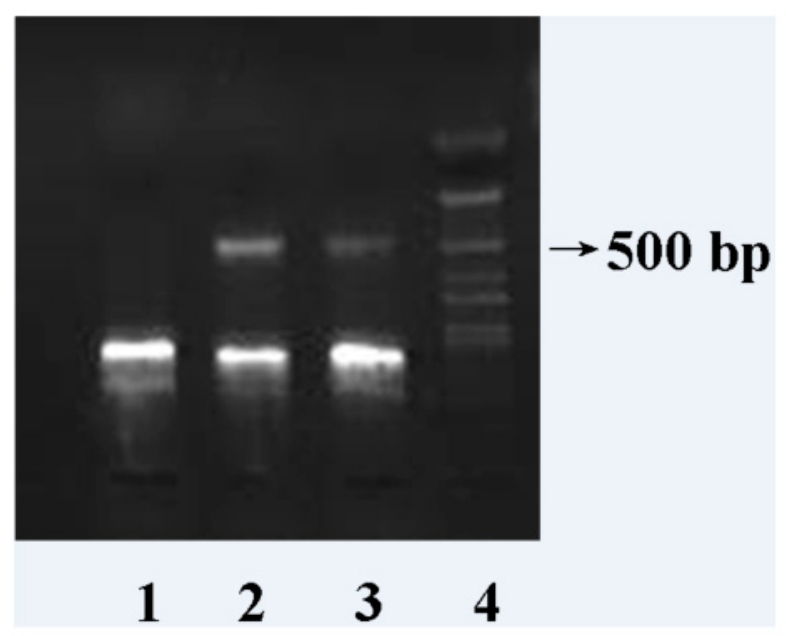

Figure 2. Restriction pattern of p15-pcDNA3.1 with EcoRI and HindIII. Lane 1 = control vector; lane 2 = restriction pattern of p15-K562-pcDNA3.1 (about $465 \mathrm{bp}$ ); lane 3 = restriction pattern of p15-PMC-pcDNA3.1 (about 465 bp); lane 4 = DGL2000 DNA marker.

However, the DNA fragment amplified from K562 cells had a 7-bp deletion from the 183 to 190 site by BLAST analysis (Figure 3).

\section{Effect of specific siRNA molecules on bcr-abl fusion gene expression}

In the RNA interference group, bcr-abl fusion gene expression was significantly lower than the control groups (Figure 4). 


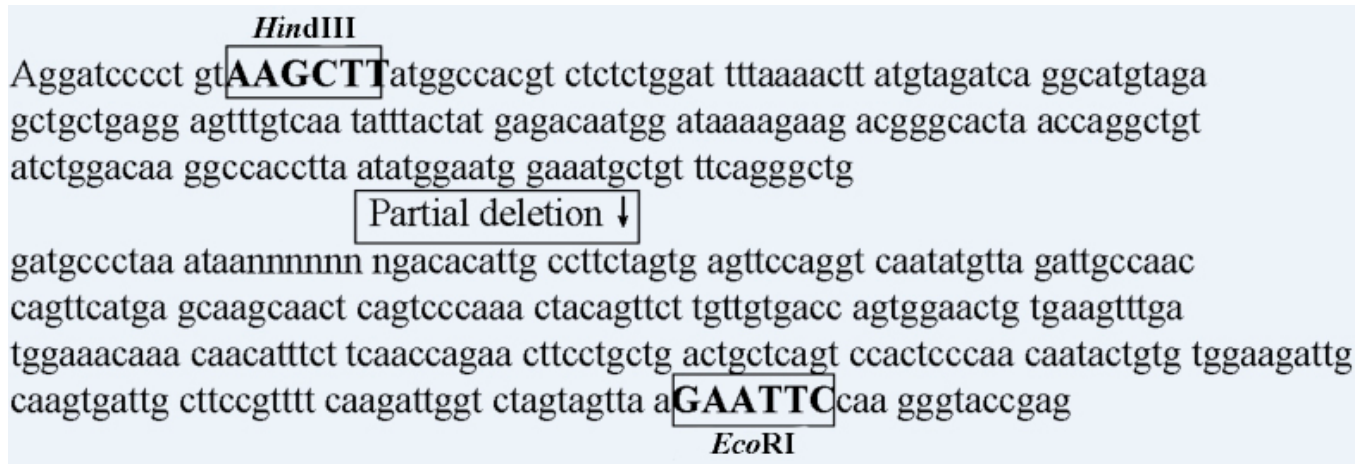

Figure 3. Sequence of the P15 gene in p15-K562-pcDNA3.1 vector (partial deletion of the p15 gene in K562).

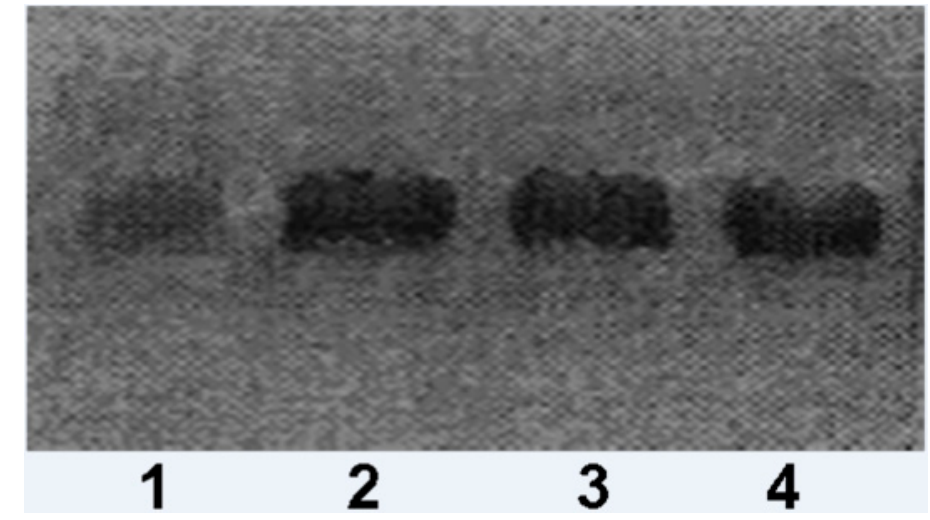

Figure 4. Bcr-abl fusion gene tested by Northern blot. Lane 1 = siRNA group; lane 2 = uncorrelated control group; Lane 3 = Lipofectamine control group; lane $4=$ normal control group.

\section{Western blot}

The cell line K562-P21-pcDNA3.1 was established and showed high expression of the p15 protein by Western blot analysis, and low expression of the cyclin D1 protein, with protein quantification and $\beta$-actin as the internal reference (Figure 5).

In the RNA interference group, the bcr-abl fusion protein and Bcl-xL were significantly decreased (Figure 5).

\section{Cell cycle}

\section{RNA interference combined with p15 gene cloning}

By flow cytometry analysis, K562 cells in the normal control group showed a high S-phase character, where the proportions of the different phases were $30.2 \% \mathrm{G} 1,57.4 \% \mathrm{~S}$ and $12.3 \% \mathrm{G} 2 / \mathrm{M}$. The cycle distribution after the transfection with p15-pcDNA3.1 plasmid for 48 


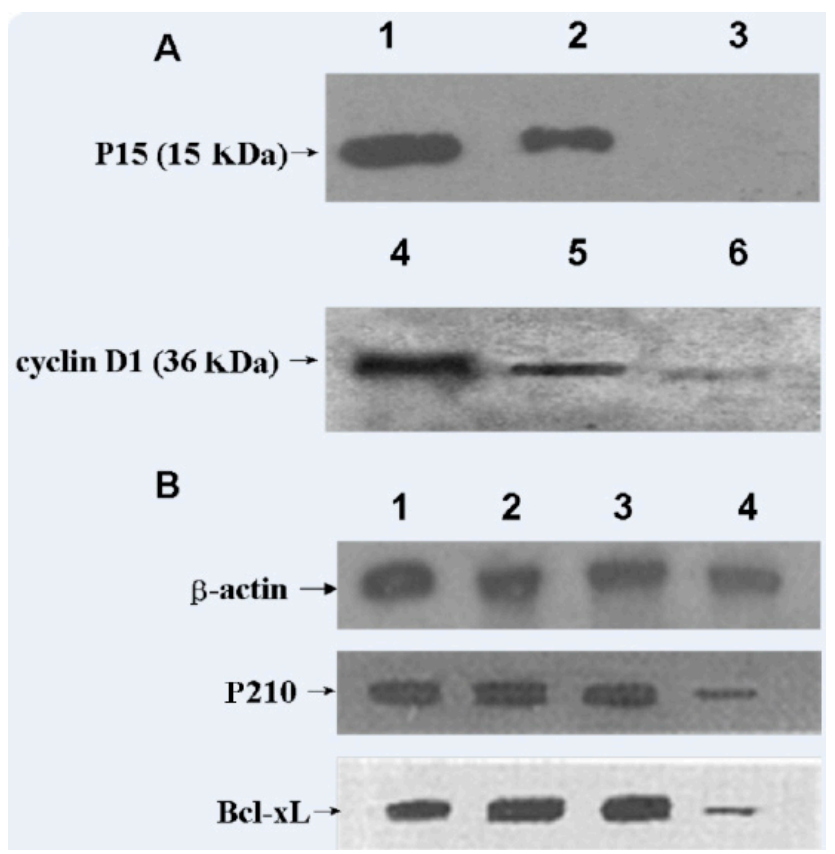

Figure 5. Expressions of p15, cyclin D1, bcr-abl and Bcl-xL protein observed by Western blot. A. Lane 1 = positive control of p15; lane 2 = positive expression of $\mathrm{p} 15$ in p15-pcDNA3.1-K562 cells; lane 3 = negative control of p15; lane 4 = positive control of cyclinD1; lane $5=$ positive expression of cyclin D1 in K562 cells; lane $6=$ decrease of cyclin D1 in p15-pcDNA3.1-K562 cells. B. Lane $1=$ normal control group; lane 2 = uncorrelated control group; lane 3 = Lipofectamine control group; lane $4=$ siRNA group.

$\mathrm{h}$ was $52.5 \% \mathrm{G} 1,38.6 \% \mathrm{~S}$ and $8.9 \% \mathrm{G} 2 / \mathrm{M}$. In the RNA interference group, the percentage of G1-phase K562 cells was significantly higher than in the control group (55.2\%). In the combination group, G1-phase was significantly higher (72.5\%) and S-phase was significantly lower (13.3\%) compared with the interference group and the p15 gene group (Figure 6).

\section{STI571 combined with p15 gene cloning}

S-phase of K562 cells was $20.3 \%$ after inducing cells with $0.25 \mu \mathrm{M}$ STI571 for $48 \mathrm{~h}$, and S-phase was decreased (13.3\%) in K562 cells transfected by 15-pcDNA3.1 recombinant vector at the same concentration of STI571 (Figure 6).

\section{Apoptosis results}

\section{RNA interference combined with p15 gene cloning}

The $\mathrm{p} 15$ gene alone transfection did not show an apoptotic peak. The apoptosis rate was about $15.4 \%$ after RNA interference alone for $24 \mathrm{~h}$. In the combination group of RNA interference and p15 gene transfection, apoptotic cells were significantly increased $(31.4 \%$ apoptotic rate) compared with the RNA interference group (Figure 6). 


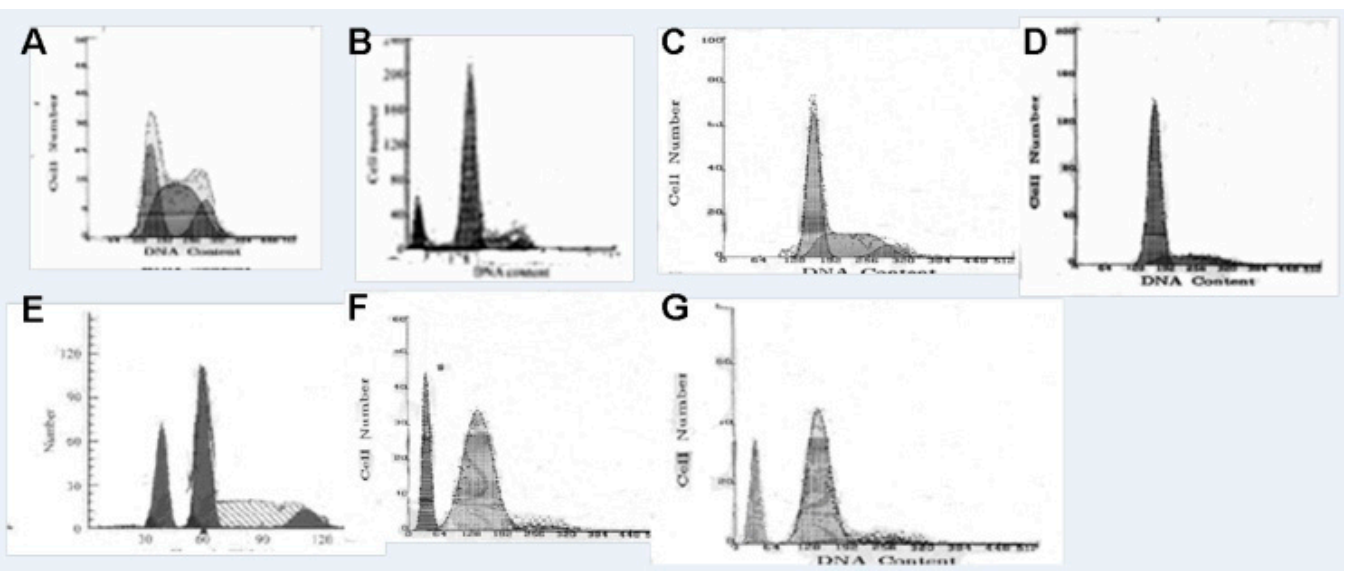

Figure 6. Cell cycle and apoptosis of K562 transfected by siRNA and STI571 and p15. A. Cell cycle of control K562 cells (S-phase, 59.6\%). B. Cell cycle of K562 cells transfected by siRNA (apoptosis rate, $15.4 \%$; G1-phase, $55.2 \%$ ). C. Cell cycle of K562 cells transfected by p15 gene clone (G1-phase, 52.5\%; S-phase, $38.6 \%$ ). D. Cell cycle of K562 cells affected by $0.25 \mu \mathrm{M}$ STI571 (S-phase $20.3 \%$ ). E. Cell cycle and apoptosis of K562 cells transfected by siRNA and p15 (apoptosis rate, 31.4\%; G1-phase, 72.5\%; S-phase, 13.3\%). F. Cell cycle and apoptosis of K562 cells affected by STI571 and p15 (apoptosis rate, $28.4 \%$; S-phase, $13.3 \%$ ). G. Cell cycle and apoptosis of K562 cells transfected by siRNA and STI571 (apoptosis rate, 27.2\%; S-phase, 12.7\%).

\section{STI571 combined with p15 gene cloning}

The p15 gene transfection group did not show an apoptotic peak, K562 cells induced with $0.25 \mu \mathrm{M}$ STI571 for $48 \mathrm{~h}$ did not show an apoptotic peak, and a significant apoptotic peak was seen (28.4\% apoptotic rate) in K562 cells transfected with 15-pcDNA3.1 recombinant vector at the same concentration of STI571 for $48 \mathrm{~h}$ (Figure 6).

\section{RNA interference combined with STI571}

In the cell proliferation observed by ${ }^{3} \mathrm{H}-\mathrm{TdR}$ incorporation, there was no significant difference in the pre-experiment of K562 cells induced with 30,60, 120 pM siRNA, which suggested the $60 \mathrm{pM}$ siRNA was sufficient for interference effects without a dose-dependent effect, including the effects on cell proliferation and apoptosis. However, the effect of STI571 on cell proliferation and apoptosis showed a dose-dependent character in a certain concentration range. In the pre-experiment, STI571 at a concentration of $0.1,0.25,0.5,1,2.5,5$, and 10 $\mu \mathrm{M}$ showed cell proliferation inhibition and apoptosis induction in a dose-dependent manner. However, at a certain STI571 concentration, the proportion of apoptosis did not appear to increase significantly. The proportion of apoptosis did not show a significant increase at $60 \mathrm{pM}$ siRNA combined with $5 \mu \mathrm{M}$ STI571, but we found that the apoptotic proportion was increased at $30 \mathrm{pM}$ siRNA combined with $0.25 \mu \mathrm{M}$ STI571 compared to the siRNA group (Figure 6).

\section{Analysis of cell survival rate}

MTT results showed that the K562 cell proliferation rate was high, and its population 
doubling time was about 18-24 h. In K562 cells transfected by pcDNA3.1 empty vector, there was no significant difference in cell proliferation rate compared with the control group. K562 cells transfected with p15-pcDNA3.1 recombinant plasmid showed a significantly slower proliferation rate than the control group, and its population doubling time was about $48-60 \mathrm{~h}$.

The combination group of RNA interference and p15-pcDNA3.1 transfection showed lower cell survival rate compared with both the RNA interference group and p15-pcDNA3.1 transfection group (Figure 7). The combination group of STI571 and p15-pcDNA3.1 transfection showed lower cell survival rate compared with both the STI571 group and $p$ 15-pcDNA3.1 transfection group (Figure 8).

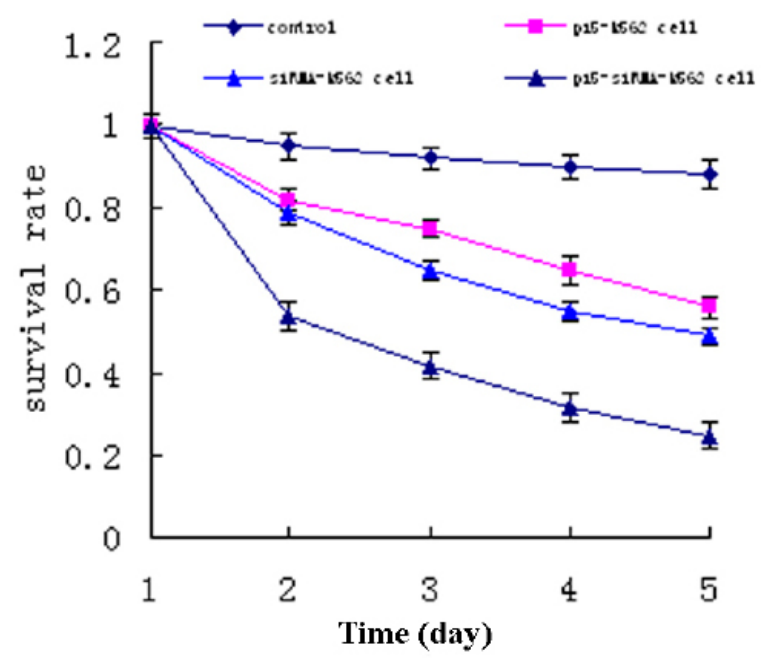

Figure 7. Growth curves of K562 transfected by siRNA and p15 (cooperation function in proliferation inhibition to K562 cell by siRNA and p15).

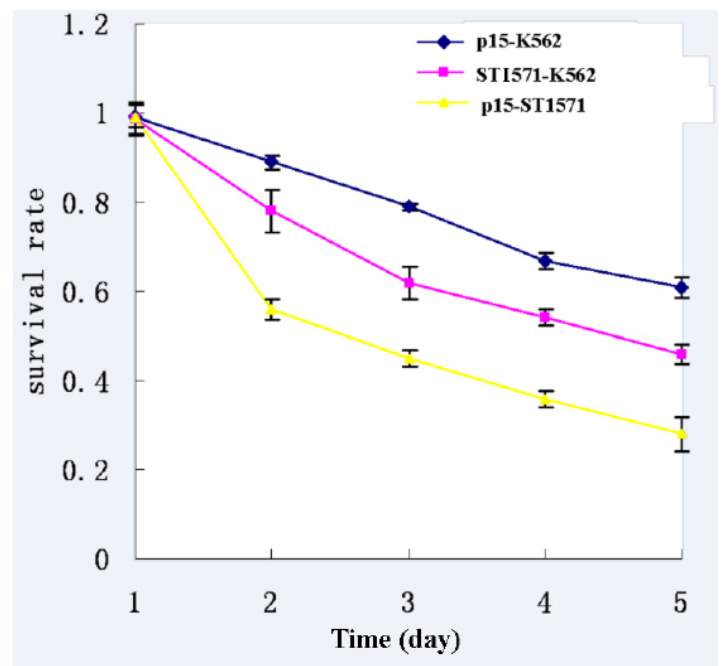

Figure 8. Growth curves of K562 transfected by STI571 and p15. 


\section{DISCUSSION}

Molecular-targeted therapy drug of STI571 and specific RNA interference technology had a milestone in the treatment of CML (Zaree-Mahmodabady et al., 2010; Jain and van Besien, 2011), even expressing a trend to replace bone marrow transplant. With the further research, it was found that CML cells would gradually develop drug-resistant mechanisms against the above treatments. Thus, multi-drug or multi-method combination will be a good development trend in the treatment of CML (Druker et al., 2002). p15, with the regulation of cell cycle and the suppression of tumor, has played an important role in the development of CML, and had been found to show abnormal expression or inactivation in many tumors (Güran et al., 1998). In the present study, we applied the combination of different treatments, namely specific siRNA inhibition of the bcr-abl fusion gene, STI571 inhibiting p210 protein activity and p15 gene expression by cloning, which was expected to play an important role in cell cycle regulation and tumor suppression for better curative effect.

The study found that siRNA showed a strong inhibition of the bcr-abl fusion gene, and induced apoptosis in K562 cells. Meanwhile, the RNA interference group showed significant G1-phase arrest in K562 cells, suggesting that DNA synthesis was blocked in K562 cells, even developing apoptosis under the specific siRNA. It was confirmed that specific siRNA played a role in inhibiting cell proliferation and inducing apoptosis in K562 cells. However, the effect of signal siRNA did not make the bcr-abl fusion gene completely silent, and the proportion of apoptosis induction was not enough for the treatment of CML. On the basis of the different pathogenesis, combination therapy should be applied in the treatment of CML. In the present study, the $\mathrm{p} 15$ gene, as a tumor suppressor was combined with specific siRNA, which achieved good effects against CML.

The p15 gene is a member of the cyclin-dependent kinase inhibitor Ink4 family (inhibitor of CDK4), which encodes one of the CDK inhibitor protein family, specifically inhibiting the phosphorylation kinase of cyclin D1-CDK4/6 complex (Bies et al., 2010).

The p15 gene showed genetic deletion, mutation, and methylation inactivation in leukemia cells, and its deletion, mutation and inactivation were more frequent in acute lymphoblastic leukemia, but scarce in myeloid leukemia. In most CML patients, p15 gene abnormality has been rarely detected (Bleichert et al., 2001; Kusy et al., 2004). Even if the acute transformation to leukomonocytes appeared in CML patients, p15 gene absence was still scarce (Bleichert et al., 2001). In the present study, repeated sequence confirmed that the p15 gene has seven consecutive base deletions in K562 cells, and p15 gene expression was present in CML acute transformation to medullary system, because K562 was cell line of CML transformed into erythroleukemia. Importantly, its absent sites were in the middle of the p 15 cDNA sequence, resulting in a completely different amino acid sequence as that of the p15 protein, and denatured p15 protein lost the ability to inhibit CDK4/CDK6 activity. So it could be inferred that the lack of normal p15 protein was one reason for malignant proliferation of K562 cells. The transfection of exogenous p15 gene into K562 cells would be helpful to regulate cell cycle and inhibit the proliferation of malignant cell by replacing abnormal p15 protein.

In view of p15 gene specificity, shorter than p53, p21, p27 in the length, etc., direct effect on cell cycle initiation factor of cyclin D1-CDK4/CDK6 complex, rapid effectiveness, the transfection of the exogenous p15 gene into cell lines with $\mathrm{p} 15$ gene mutation could have an anti-leukemic effect, leading to a hot spot of gene therapy for leukemia. The combination 
of RNA interference, STI571 and p15 gene therapy was a new beneficial attempt for CML management. The present study found that the transfection of the p15 gene into K562 cells combined with RNA interference and STI571 showed a synergistic effect in inhibiting cell proliferation and inducing apoptosis. At present, we believe that the p15 gene has a similar function as the p16 gene, positively correlated with cell cycle arrest, proliferation inhibition and apoptosis. p15 gene expression can arrest cells in G1-phase, significantly decreasing S-phase cells, inhibiting cell proliferation and accelerating apoptosis (Fuxe et al., 2000). The p15 gene is a downstream gene regulated by the bcr-abl fusion gene (Kusy et al., 2004), which can downregulate the p15 protein and mRNA levels through the PI3K pathway. Also, RNA interference and STI571 cannot only prevent $\mathrm{p} 15$ suppression induced by bcr-abl, but also induce $\mathrm{p} 15$ protein expression, via a mechanism involving the inhibition of the PI3K signal pathway and the decrease of $\mathrm{p} 15$ protein degradation. At the same time, STI571 can inhibit the expression of a variety of cell cycle factors, such as cyclin D1 and cyclin E, etc., which attenuate the inhibition of the p15 protein, cyclin D1 being an inhibitor of the p15 protein. Therefore, the 15 gene transfection could rebuild the cell cycle pathway in K562 cells, which would strengthen the apoptosis of K562 cells and show a synergistic effect by combining with RNA interference and STI571.

Our study found that regular doses of siRNA did not show an increase in apoptosis proportion when combined with STI571, which was consistent with a previous study (Wilda et al., 2002). We believe that the possible mechanism was the strong apoptosis induction of STI571, where a regular dose could induce significant apoptosis, and where a dose increase or the addition of new factors would not promote apoptosis. However, a small dose of $30 \mathrm{pM}$ siRNA combined with $0.25 \mu \mathrm{M}$ STI571 appeared significantly increase apoptosis proportion compared with the two agents alone. The above results suggested that the combination of lowdose RNA interference and STI571 displayed a synergistic effect before reaching saturation effect concentration, but specific range and law still need further research.

In summary, siRNA alone could not make the bcr-abl fusion gene completely silent, and the combination of RNA interference with $\mathrm{p} 15$ gene cloning led to significant reduction in cell proliferation. The same effect, meanwhile, was displayed in the combination of STI571 with the p15 gene. Low-dose RNA interference combined with STI571 appeared to have a synergistic effect in the induction of apoptosis. Our study confirmed that the combined application of RNA interference, STI571 and the p15 gene could achieve better efficacy, providing a useful attempt for multi-gene-targeted therapy in CML.

\section{REFERENCES}

Bengió RM, Riva ME, Moiraghi B, Lanari E, et al. (2011). Clinical outcome of chronic myeloid leukemia imatinibresistant patients: do BCR-ABL kinase domain mutations affect patient survival? First Multicenter Argentinean Study. Leuk. Lymphoma 52: 1720-1726.

Bies J, Sramko M, Fares J, Rosu-Myles M, et al. (2010). Myeloid-specific inactivation of p15Ink4b results in monocytosis and predisposition to myeloid leukemia. Blood 116: 979-987.

Bleichert A, Fiedler W, Claussen U, Ernst G, et al. (2001). A long distance-PCR derived FISH probe detects a deletion between p15 and p16 in CML and T-ALL patients. Int. J. Mol. Med. 7: 591-595.

Druker BJ, O'Brien SG, Cortes J and Radich J (2002). Chronic myelogenous leukemia. Hematolol. Am. Soc. Hematol. Educ. Program. 111-135.

Elbashir SM, Harborth J, Lendeckel W, Yalcin A, et al. (2001). Duplexes of 21-nucleotide RNAs mediate RNA interference in cultured mammalian cells. Nature 411: 494-498.

Fuxe J, Akusjarvi G, Goike HM, Roos G, et al. (2000). Adenovirus-mediated overexpression of p15INK4B inhibits human glioma cell growth, induces replicative senescence, and inhibits telomerase activity similarly to p16INK4A. Cell 
Growth Differ. 11: 373-384.

Güran S, Bahce M, Beyan C, Korkmaz K, et al. (1998). P53, p15INK4B, p16INK4A and p57KIP2 mutations during the progression of chronic myeloid leukemia. Haematologia 29: 181-193.

Helgason GV, Karvela M and Holyoake TL (2011). Kill one bird with two stones: potential efficacy of BCR-ABL and autophagy inhibition in CML. Blood 118: 2035-2043.

Jain N and van Besien K (2011). Chronic myelogenous leukemia: role of stem cell transplant in the imatinib era. Hematol. Oncol. Clin. North Am. 25: 1025-48.

Kusy S, Larsen CJ and Roche J (2004). p14ARF, p15INK4b and p16INK4a methylation status in chronic myelogenous leukemia. Leuk. Lymphoma 45: 1989-1994.

Leonetti F, Stefanachi A, Nicolotti O, Catto M, et al. (2011). BCR-ABL inhibitors in chronic myeloid leukemia: process chemistry and biochemical profile. Curr. Med. Chem. 18: 2943-2959.

Nguyen TT, Mohrbacher AF, Tsai YC, Groffen J, et al. (2000). Quantitative measure of c-abl and p15 methylation in chronic myelogenous leukemia: biological implications. Blood 95: 2990-2992.

Nicolini FE, Masszi T, Shen Z, Gallagher NJ, et al. (2011). Expanding nilotinib access in clinical trials (ENACT), an open-label, multicenter study of oral nilotinib in adult patients with imatinib-resistant or -intolerant chronic myeloid leukemia in the accelerated phase or blast crisis. Leuk. Lymphoma 24.

Swords R, Mahalingam D, Padmanabhan S, Carew J, et al. (2009). Nilotinib: optimal therapy for patients with chronic myeloid leukemia and resistance or intolerance to imatinib. Drug Des. Devel. Ther. 3: 89-101.

Wilda M, Fuchs U, Wossmann W and Borkhardt A (2002). Killing of leukemic cells with a BCR/ABL fusion gene by RNA interference (RNAi). Oncogene 21: 5716-5724.

Yamauchi T and Ueda T (2011). Nilotinib as a second-line treatment for chronic myeloid leukemia. Gan To Kagaku Ryoho 38: 911-915.

Zaree-Mahmodabady A, Javadi HR, Kamali M, Najafi A, et al. (2010). Bcr-abl silencing by specific small-interference RNA expression vector as a potential treatment for chronic myeloid leukemia. Iran. Biomed. J. 14: 1-8. 